\title{
Geometric-arithmetic averaging of dyadic weights
}

\author{
Jill Pipher, Lesley A. Ward and Xiao Xiao
}

\begin{abstract}
The theory of Muckenhoupt's weight functions arises in many areas of analysis, for example in connection with bounds for singular integrals and maximal functions on weighted spaces. We prove that a certain averaging process gives a method for constructing $A_{p}$ weights from a measurably varying family of dyadic $A_{p}$ weights. This averaging process is suggested by the relationship between the $A_{p}$ weight class and the space of functions of bounded mean oscillation. The same averaging process also constructs weights satisfying reverse Hölder $\left(R H_{p}\right)$ conditions from families of dyadic $R H_{p}$ weights, and extends to the polydisc as well.
\end{abstract}

\section{Introduction}

Several classes of functions are defined in terms of a property that the function must satisfy on each interval, with a uniform constant. Well known examples from harmonic analysis and complex analysis include Muckenhoupt's $A_{p}$ weights, the reverse-Hölder weight classes $R H_{p}$, the class of doubling weights, and the space BMO of functions of bounded mean oscillation. Such classes have strictly larger dyadic analogues, where the defining property is required only on dyadic intervals. Certain types of averaging provide a bridge between these dyadic counterparts and the original function classes. Specifically, these averages convert each suitable family of functions in the dyadic class to a single function in the smaller, nondyadic class. We can think of averaging as an improving operation, in this sense.

2000 Mathematics Subject Classification: Primary: 42B35; Secondary: 42B25.

Keywords: Dyadic harmonic analysis, multiparameter harmonic analysis, dyadic weights, bounded mean oscillation, Muckenhoupt weights, $A_{p}$ weights, reverse Hölder weights, product weights, bidisc, polydisc. 
An easily stated example is the following. If all translates of a function $f$ defined on the unit circle $\mathbb{T}=[0,1]$ are in dyadic BMO, or equivalently if $f$ is in dyadic BMO on every translated grid of dyadic intervals on the circle, then the function $f$ itself is in true BMO. This result is a special case of a theorem in [5], applied to the identity

$$
f(x)=\int_{0}^{1} \tau_{t} f(x+t) d t,
$$

where for $t \in \mathbb{R}$ the translation operator $\tau_{t}$ is defined by $\tau_{t} f(\cdot):=f(\cdot-t)$, and $x+t$ is to be interpreted as $x+t \bmod 1$.

Now, what if a function $f$ on $\mathbb{T}$ can be written as the translation-average

$$
f(x):=\int_{0}^{1} f^{t}(x+t) d t
$$

of dyadic BMO functions $\left\{f^{t}\right\}_{t \in[0,1]}$ that are not identical translates of each other? If they satisfy the hypotheses of [5], then still $f$ is in true BMO. However, the analogous statements can fail for $A_{p}$ weights, for $R H_{p}$ weights, and for doubling weights [16].

In this paper we show that a different type of averaging works for both $A_{p}$ and $R H_{p}$ (Theorems 1 and 2). This is the geometric-arithmetic average defined by

$$
\Omega(x):=\exp \left\{\int_{0}^{1} \log \omega^{t}(x+t) d t\right\}
$$

where $\left\{\omega^{t}\right\}_{t \in[0,1]}$ is a suitable family of weights in $A_{p}^{d}$ or $R H_{p}^{d}$.

We also observe that translation-averaging does work for $A_{p}$ and also for $R H_{p}$, under the additional assumption that the functions $\omega^{t}$ are doubling weights, not just dyadic doubling weights (Theorem 3). Equivalently, the translation-average of $A_{p}$ weights is an $A_{p}$ weight, and similarly for $R H_{p}$.

All these results generalize to the polydisc (Theorems 4 and 5).

The paper is organized as follows. In Section 2, we state our geometricarithmetic averaging results on the circle. In Section 3, we collect the definitions and background results used in the paper. Also, Lemma 1 in that section gives a unified characterization of weights in $A_{p}$ for $1 \leq p \leq \infty$, $R H_{p}$ for $1<p \leq \infty$, and their dyadic counterparts, in terms of conditions on the oscillation of their logarithms. We take some care throughout in tracing the dependence of the various constants, although some of our bounds on the constants may not be sharp. In Section 4, we prove geometric-arithmetic averaging for $A_{p}^{d}$ and $R H_{p}^{d}$ weights (Theorems 1 and 2). In Section 5, we prove translation-averaging for $A_{p}^{d}$ and $R H_{p}^{d}$ weights that are doubling (Theorem 3). In Section 6, we generalize our results to the polydisc (Theorems 4 and 5). 


\section{The geometric-arithmetic average on the circle}

In [16], examples are constructed to show that, for $\left\{\omega^{t}\right\}_{t \in[0,1]}$ a measurably varying family of dyadic $A_{p}^{d}$ weights (for arbitrary $p$ with $1 \leq p \leq \infty$ ) or dyadic $R H_{p}^{d}$ weights (for arbitrary $p$ with $1<p<\infty$ ) on the circle $\mathbb{T}=[0,1]$, with uniformly bounded dyadic $A_{p}^{d}$ or $R H_{p}^{d}$ constants, the translation-average $\omega(x):=\int_{0}^{1} \omega^{t}(x+t) d t$ is not necessarily a doubling weight. Therefore $\omega$ need not be in true $A_{p}$, nor in true $R H_{p}$.

The main result of the current paper is that, by contrast, the geometricarithmetic average $\Omega(x):=\exp \left\{\int_{0}^{1} \log \omega^{t}(x+t) d t\right\}$ always turns a measurably varying family of suitably normalized dyadic $A_{p}^{d}$ weights into an $A_{p}$ weight (for arbitrary $p$ with $1 \leq p \leq \infty$ ), and a measurably varying family of suitably normalized dyadic $R H_{p}^{d}$ weights into an $R H_{p}$ weight (for arbitrary $p$ with $1<p \leq \infty)$.

Theorem 1. Fix $p$ with $1 \leq p \leq \infty$. Let $\left\{\omega^{t}\right\}_{t \in[0,1]}$ be a family of dyadic $A_{p}$ weights on the circle $\mathbb{T}, \omega^{t} \in A_{p}^{d}(\mathbb{T})$, such that

(i) the mapping $t \mapsto \omega^{t}$ is measurable,

(ii) an appropriate average of the logarithms of the weights $\omega^{t}$ is finite:

$$
\int_{0}^{1} \int_{0}^{1}\left|\log \omega^{t}(x)\right| d x d t<\infty
$$

and

(iii) the $A_{p}^{d}$ constants $A_{p}^{d}\left(\omega^{t}\right)$ are uniformly bounded, independent of $t \in[0,1]$.

Then the geometric-arithmetic average

$$
\Omega(x)=\exp \left\{\int_{0}^{1} \log \omega^{t}(x+t) d t\right\}
$$

of the dyadic weights $\omega^{t}$ belongs to $A_{p}$ on $\mathbb{T}$. Moreover, the $A_{p}$ constant of $\Omega$ depends only on $p$ and on the bound on the $A_{p}^{d}$ constants of the $\omega^{t}$.

Remark. A simple heuristic motivation for this result is as follows. The weights $\omega^{t}$ belong to $A_{p}^{d}$, so their logarithms $\log \omega^{t}$ are in $\mathrm{BMO}_{d}$. Therefore, as shown in [5] (and later [13] for the one- and two-parameter settings and [15] for the general multiparameter setting), the translation-average $\log \Omega$ of the functions $\log \omega^{t}$ is in BMO, and so by the John-Nirenberg Theorem [8] sufficiently small powers $\Omega^{\delta}$ of $\Omega$ are in $A_{p}$. It remains to show that $\Omega$ itself is in $A_{p}$.

Hypothesis (ii) of the theorem is merely a normalization condition: the fact that each $\omega^{t}(x)$ belongs to $A_{p}^{d}$ already implies that $\log \omega^{t}(x)$ belongs to $L^{1}(d x)$. 
The analogous result to Theorem 1 holds for reverse-Hölder weights.

Theorem 2. Fix $p$ with $1<p \leq \infty$. Let $\left\{\omega^{t}\right\}_{t \in[0,1]}$ be a family of dyadic $R H_{p}$ weights on the circle $\mathbb{T}, \omega^{t} \in R H_{p}^{d}(\mathbb{T})$, such that hypotheses $(i)$ and $(i i)$ of Theorem 1 hold, and

(iii') the $R H_{p}^{d}$ constants $R H_{p}^{d}\left(\omega^{t}\right)$ are uniformly bounded, independent of $t \in[0,1]$.

Then the geometric-arithmetic average $\Omega(x)=\exp \left\{\int_{0}^{1} \log \omega^{t}(x+t) d t\right\}$ of the dyadic weights $\omega^{t}$ lies in $R H_{p}$ on $\mathbb{T}$. The $R H_{p}$ constant of $\Omega$ depends only on $p$ and on the bound on the $R H_{p}^{d}$ constants of the $\omega^{t}$.

These results also hold on $\mathbb{T}^{k}$, with constants that depend on the dimension $k$. In addition, they hold in the setting of the polydisc; see Section 6 .

Remark. It is not necessary for the integral in $t$ to be taken over the whole interval $[0,1]$. The proofs below go through without change when the integral is taken over an arbitrary subset $E \subset[0,1]$ of positive measure.

\section{Definitions and tools}

In this section we collect useful material about doubling weights, the weight classes $A_{p}$ and $R H_{p}$, and their relationship to BMO. For fuller accounts of the theory of $A_{p}$ and $R H_{p}$ weights, see for example [3], [4], [6], and [2].

Let $\mathbb{T}$ denote the unit circle, obtained by identifying the endpoints of the interval $[0,1]$. In the definitions of our averages $\Omega$ and $\omega, x+t$ is to be interpreted as $x+t \bmod 1$.

Denote the collection of dyadic subintervals $I$ of the circle $\mathbb{T}$ by $\mathcal{D}=\mathcal{D}[0,1]$ :

$$
\mathcal{D}:=\{[0,1]\} \cup\left\{I=\left[\frac{j}{2^{k}}, \frac{j+1}{2^{k}}\right) \mid k \in \mathbb{N}, j \in\left\{0,1, \ldots, 2^{k}-1\right\}\right\} .
$$

Throughout the paper, $Q$ denotes a general subinterval of $\mathbb{T}$, while $I, J, K$ and $L$ denote dyadic subintervals of $\mathbb{T}$.

The functions we consider are real-valued.

We use the symbol $|E|$ to denote the Lebesgue measure of a set $E$, the symbol $f_{E}$ to denote $\frac{1}{|E|} \int_{E}$, and the symbol $f_{E}$ for the average value $f_{E} f$ of a function $f$ on a set $E$. The notation $E \subset F$ includes the possibility $E=F$.

Definition 1. Let $\omega(x)$ be a nonnegative locally integrable function on the circle $\mathbb{T}$. We say $\omega$ is a doubling weight with doubling constant $C$ if for all intervals $Q \subset \mathbb{T}$

$$
\int_{\widetilde{Q}} \omega(x) d x \leq C \int_{Q} \omega(x) d x
$$


where $\widetilde{Q}$ is the double of $Q$; that is, $\widetilde{Q}$ is the interval with the same midpoint as $Q$ and twice the length of $Q$. We say $\omega$ is a dyadic doubling weight with dyadic doubling constant $C$ if the analogous inequality holds for all dyadic intervals $I \subset \mathbb{T}$, where $\widetilde{I}$ is the dyadic double of $I$ : that is, $\widetilde{I}$ is unique dyadic interval of length $|\widetilde{I}|=2|I|$ that contains $I$.

The $A_{p}$ weights were identified by Muckenhoupt [12] as the weights $\omega$ for which the Hardy-Littlewood maximal function is bounded from $L^{p}(d \mu)$ to itself, where $d \mu=\omega(x) d x$. Here we give the definitions of the classes $A_{p}$ and $R H_{p}$ on the circle $\mathbb{T}$; the analogous definitions hold on $\mathbb{R}$ and on (oneparameter) $\mathbb{R}^{k}$. We delay the corresponding definitions for the polydisc setting until Section 6 .

Definition 2. Let $\omega(x)$ be a nonnegative locally integrable function on the circle $\mathbb{T}$. For real $p$ with $1<p<\infty$, we say $\omega$ is an $A_{p}$ weight, written $\omega \in A_{p}$, if

$$
A_{p}(\omega):=\sup _{Q}\left(f_{Q} \omega\right)\left(f_{Q}\left(\frac{1}{\omega}\right)^{1 /(p-1)}\right)^{p-1}<\infty .
$$

For $p=1$, we say $\omega$ is an $A_{1}$ weight, written $\omega \in A_{1}$, if

$$
A_{1}(\omega):=\sup _{Q}\left(f_{Q} \omega\right)\left(\frac{1}{\operatorname{ess~inf}_{x \in Q} \omega(x)}\right)<\infty .
$$

For $p=\infty$, we say $\omega$ is an A-infinity weight, written $\omega \in A_{\infty}$, if

$$
A_{\infty}(\omega):=\sup _{Q}\left(f_{Q} \omega\right) \exp \left(f_{Q} \log \left(\frac{1}{\omega}\right)\right)<\infty .
$$

Here the suprema are taken over all intervals $Q \subset \mathbb{T}$. The quantity $A_{p}(\omega)$ is called the $A_{p}$ constant of $\omega$.

The dyadic $A_{p}$ classes $A_{p}^{d}$ for $1 \leq p \leq \infty$ are defined analogously, with the suprema $A_{p}^{d}(\omega)$ being taken over only the dyadic intervals $I \subset \mathbb{T}$.

Definition 3. Let $\omega(x)$ be a nonnegative locally integrable function on the circle $\mathbb{T}$. For real $p$ with $1<p<\infty$, we say $\omega$ is a reverse-Hölder-p weight, written $\omega \in R H_{p}$ or $\omega \in B_{p}$, if

$$
R H_{p}(\omega):=\sup _{Q}\left(f_{Q} \omega^{p}\right)^{1 / p}\left(f_{Q} \omega\right)^{-1}<\infty .
$$

For $p=\infty$, we say $\omega$ is a reverse-Hölder-infinity weight, written $\omega \in R H_{\infty}$ or $\omega \in B_{\infty}$, if

$$
R H_{\infty}(\omega):=\sup _{Q}\left(\operatorname{ess}_{x \in Q} \sup \omega(x)\right)\left(f_{Q} \omega\right)^{-1}<\infty .
$$

Here the suprema are taken over all intervals $Q \subset \mathbb{T}$. The quantity $R H_{p}(\omega)$ is called the $R H_{p}$ constant of $\omega$. 
For $1<p \leq \infty$, we say $\omega$ is a dyadic reverse-Hölder-p weight, written $\omega \in R H_{p}^{d}$ or $\omega \in B_{p}^{d}$, if the analogous condition

$$
\sup _{I \in \mathcal{D}}\left(f_{I} \omega^{p}\right)^{1 / p}\left(f_{I} \omega\right)^{-1}<\infty \quad \text { or } \sup _{I \in \mathcal{D}}(\operatorname{ess} \sup \omega(x))\left(f_{I} \omega\right)^{-1}<\infty
$$

holds with the supremum being taken over only the dyadic intervals $I \subset \mathbb{T}$, and if in addition $\omega$ is a dyadic doubling weight. We define the $R H_{p}^{d}$ constant $R H_{p}^{d}(\omega)$ of $\omega$ to be the larger of this dyadic supremum and the dyadic doubling constant.

The $A_{p}$ inequality (or the $R H_{p}$ inequality) implies that the weight $\omega$ is doubling, and the dyadic $A_{p}$ inequality implies that $\omega$ is dyadic doubling. However, the dyadic $R H_{p}$ inequality does not imply that $\omega$ is dyadic doubling, which is why the dyadic doubling assumption is needed in the definition of $R H_{p}^{d}$.

The $A_{p}$ classes are nested and increasing with $p$, while the $R H_{p}$ classes are nested and decreasing with $p$. Moreover,

$$
A_{q}(\omega) \leq A_{p}(\omega), \quad \text { for } 1 \leq p<q \leq \infty,
$$

and

$$
R H_{p}(\omega) \leq R H_{q}(\omega), \quad \text { for } 1<p<q \leq \infty
$$

Also

$$
A_{\infty}=\bigcup_{p \geq 1} A_{p}=\bigcup_{q>1} R H_{q}, \quad A_{1} \subsetneq \bigcap_{p>1} A_{p}, \quad R H_{\infty} \subsetneq \bigcap_{p>1} R H_{p} .
$$

The dyadic versions of the assertions in this paragraph also hold.

The example $w(x)=[\log (1 /|x|)]^{-1}$ (for $x$ near zero) cited in [9] shows that the inclusion of $A_{1}$ in $\bigcap_{p>1} A_{p}$ is proper.

The example $\omega(x)=\max \{\log (1 /|x|), 1\}$ given in [2] shows that $R H_{\infty}$ is a proper subset of $\cap_{p>1} R H_{p}$.

However, as noted in [2], if a weight $\omega$ is in $A_{p}$ for each $p>1$ and if the constants $A_{p}(\omega)$ are uniformly bounded, then $\omega \in A_{1}$; and the corresponding statement holds for $R H_{p}$ and $R H_{\infty}$.

As noted above, for a nonnegative locally integrable function $\omega$,

$$
\begin{aligned}
\omega \text { is in } A_{\infty} & \Longleftrightarrow \omega \text { is in } A_{p} \text { for some } p \in[1, \infty) \\
& \Longleftrightarrow \omega \text { is in } R H_{q} \text { for some } q \in(1, \infty) .
\end{aligned}
$$

In the first equivalence the $A_{\infty}$ constant depends only on the $A_{p}$ constant and on $p$, which in turn depend only on the $A_{\infty}$ constant. Similarly, the $A_{\infty}$ 
constant depends only on the $R H_{q}$ constant and on $q$, which depend only on the $A_{\infty}$ constant. See for example [6, Theorem 9.3.3] where the constants in these and other characterizations of $A_{\infty}$ are carefully analyzed. The analogous statements hold for the dyadic classes $A_{\infty}^{d}, A_{p}^{d}$, and $R H_{p}^{d}$.

The classes of $A_{p}$ and $R H_{p}$ weights can be characterized by conditions on the oscillation of the logarithm of the weight, as follows.

Lemma 1. Let $\omega$ be a nonnegative locally integrable function on $\mathbb{T}$. Let $\varphi:=\log \omega$. Then the following five statements hold.

(a) $\omega$ is in $A_{\infty}$ if and only if

$$
\sup _{Q} f_{Q} \exp \left\{\varphi(x)-\varphi_{Q}\right\} d x<\infty
$$

(b) For $1<p<\infty, \omega$ is in $A_{p}$ if and only if inequality (3.1) holds and also

$$
\sup _{Q} f_{Q} \exp \left\{\frac{-\left(\varphi(x)-\varphi_{Q}\right)}{p-1}\right\} d x<\infty
$$

(c) $\omega$ is in $A_{1}$ if and only if inequality (3.1) holds and also

$$
\sup _{Q}\left[\varphi_{Q}-\underset{x \in Q}{\operatorname{ess} \inf } \varphi(x)\right]<\infty \text {. }
$$

(d) $\omega$ is in $R H_{\infty}$ if and only if

$$
\sup _{Q}\left[\operatorname{ess} \sup _{x \in Q} \varphi(x)-\varphi_{Q}\right]<\infty .
$$

(e) For $1<p<\infty, \omega$ is in $R H_{p}$ if and only if

$$
\sup _{Q} f_{Q} \exp \left\{p\left(\varphi(x)-\varphi_{Q}\right)\right\} d x<\infty
$$

In each part, the value of $A_{p}(\omega)$ or $R H_{p}(\omega)$ depends on the value(s) of the supremum (suprema) in the characterization given and, when $1<p<\infty$, also on $p$. Conversely, the value(s) of the supremum (suprema) depend on the value of $A_{p}(\omega)$ or $R_{p}(\omega)$ and, when $1<p<\infty$, also on $p$.

Taking the suprema in inequalities (3.1)-(3.5) over only dyadic intervals $I \subset \mathbb{T}$, the dyadic analogues of parts $(a)-(e)$ hold for the dyadic classes $A_{\infty}^{d}, A_{p}^{d}, A_{1}^{d}, R H_{\infty}^{d}$, and $R H_{p}^{d}$, except that in parts $(d)$ and $(e)$ one needs in addition to inequality (3.4) or (3.5) the extra hypothesis that $\omega$ is dyadic doubling. The dependence of the constants in the dyadic case is the same as in the continuous case. 
Parts (b) and (c) appear in [4], [6], and [3], for example, and part $(d)$ is in [2, Cor 4.6]. Inequality (3.3) says that an $A_{\infty}$ weight $\omega$ lies in $A_{1}$ when its logarithm $\varphi$ belongs to the space BLO of functions of bounded lower oscillation, while inequality (3.4) says that $\omega$ is in $R H_{\infty}$ when $-\varphi$ belongs to BLO.

Proof. Let $C_{1}, C_{2}, C_{3}, C_{4}$, and $C_{5}$ be the suprema in inequalities (3.1), (3.2), (3.3), (3.4), and (3.5) respectively.

(a) It is immediate that $A_{\infty}(\omega)=C_{1}$, since for each interval $Q$ the $A_{\infty}$ quantity is

$$
\left(f_{Q} \omega(x) d x\right) \exp \left\{f_{Q} \log \frac{1}{w(x)} d x\right\}=f_{Q} \exp \left\{\varphi(x)-\varphi_{Q}\right\} d x .
$$
Let

(b) We show that $C_{1} \leq A_{p}(\omega), C_{2} \leq A_{p}(\omega)^{1 /(p-1)}$, and $A_{p}(\omega) \leq C_{1} C_{2}^{p-1}$.

$$
\psi:=\log \left[\left(\frac{1}{\omega}\right)^{1 /(p-1)}\right]=\frac{-\varphi}{p-1} .
$$

Let $Q$ be an interval in $\mathbb{T}$. Then by Jensen's inequality,

$$
\begin{aligned}
& f_{Q} \exp \left\{\varphi(x)-\varphi_{Q}\right\} d x=\left(f_{Q} w\right) \exp \left((p-1) f_{Q} \psi(x) d x\right) \\
& \leq\left(f_{Q} w\right)\left[f_{Q} \exp \psi(x) d x\right]^{p-1}=\left(f_{Q} w\right)\left[f_{Q}\left(\frac{1}{\omega}\right)^{1 /(p-1)}\right]^{p-1} \leq A_{p}(\omega) .
\end{aligned}
$$

Thus inequality (3.1) holds with $C_{1} \leq A_{p}(\omega)$. Similarly, by Jensen's inequality,

$$
\begin{array}{r}
f_{Q} \exp \left\{\frac{-\left(\varphi(x)-\varphi_{Q}\right)}{p-1}\right\} d x=\left(f_{Q}\left(\frac{1}{\omega}\right)^{1 /(p-1)}\right)\left[\exp \left(f_{Q} \varphi\right)\right]^{1 /(p-1)} \\
\leq\left[\left(f_{Q}\left(\frac{1}{\omega}\right)^{1 /(p-1)}\right)^{p-1}\left(f_{Q} \omega\right)\right]^{1 /(p-1)} \leq A_{p}(\omega)^{1 /(p-1)} .
\end{array}
$$

Thus inequality (3.3) holds with $C_{2} \leq A_{p}(\omega)^{1 /(p-1)}$.

For the converse,

$$
\begin{aligned}
\left(f_{Q} \omega\right) & \left(f_{Q}\left(\frac{1}{\omega}\right)^{1 /(p-1)}\right)^{p-1}= \\
& =\left(f_{Q} \exp \varphi(x) d x\right)\left(f_{Q} \exp \psi(x) d x\right)^{p-1} e^{-\varphi_{Q}} e^{-(p-1) \psi_{Q}} \\
& =\left(f_{Q} \exp \left\{\varphi(x)-\varphi_{Q}\right\} d x\right)\left(f_{Q} \exp \left\{\psi(x)-\psi_{Q}\right\} d x\right)^{p-1} \leq C_{1} C_{2}^{p-1},
\end{aligned}
$$

and thus $A_{p}(\omega) \leq C_{1} C_{2}^{p-1}$. 
(c) We show that $C_{1} \leq A_{1}(\omega), C_{3} \leq \log A_{1}(\omega)$, and $A_{1}(\omega) \leq C_{1} \exp C_{3}$. If $\omega$ is in $A_{1}$, then for each interval $Q$ in $\mathbb{T}$ we have

$$
f_{Q} e^{\varphi(x)} d x=f_{Q} w(x) d x \leq A_{1}(\omega) \underset{x \in Q}{\operatorname{ess} \inf } w(x) \leq A_{1}(\omega) e^{\varphi_{Q}} .
$$

It follows that

$$
f_{Q} e^{\varphi(x)-\varphi_{Q}} d x \leq A_{1}(\omega)
$$

Thus $\varphi$ satisfies inequality (3.1) with constant $C_{1} \leq A_{1}(\omega)$.

By Jensen's inequality and the $A_{1}$ property,

$$
e^{\varphi_{Q}} \leq f_{Q} w(x) d x \leq A_{1}(\omega) \exp \{\underset{x \in Q}{\operatorname{ess} \inf } \varphi(x)\}
$$

Therefore

$$
\varphi_{Q} \leq \log A_{1}(\omega)+\underset{x \in Q}{\operatorname{ess} \inf } \varphi(x) .
$$

Thus $\varphi$ satisfies inequality (3.3) with constant $C_{3} \leq \log A_{1}(\omega)$.

Now suppose that $\varphi$ satisfies inequalities (3.1) and (3.3). Then for each interval $Q$,

$$
\begin{aligned}
f_{Q} w(x) d x & =f_{Q} e^{\varphi(x)} d x \leq C_{1} e^{\varphi_{Q}} \\
& \leq C_{1} \exp \left\{C_{3}+\underset{x \in Q}{\operatorname{ess} \inf } \varphi(x)\right\}=C_{1} e^{C_{3}} \underset{x \in Q}{\operatorname{ess} \inf } w(x) .
\end{aligned}
$$

Thus $\omega$ satisfies the $A_{1}$ property with constant $A_{1}(\omega) \leq C_{1} e^{C_{3}}$.

(d) We show that $C_{4} \leq \log \left(R H_{\infty}(\omega) A_{\infty}(\omega)\right)$ and $R H_{\infty}(\omega) \leq e^{C_{4}}$, and that the bound on $C_{4}$ depends only on $R H_{\infty}(\omega)$.

Suppose $\omega$ is in $R H_{\infty}$. Then $\omega$ is in $A_{\infty}$, so inequality (3.1) holds with $C_{1}=A_{\infty}(\omega)$. Further, $\omega$ is in every $R H_{p}$ for $p \in(1, \infty)$, and $A_{\infty}(\omega)$ depends only on $R H_{p}(\omega)$, while $R H_{p}(\omega) \leq R H_{\infty}(\omega)$. Thus $A_{\infty}(\omega)$ depends only on $R H_{\infty}(\omega)$. Now for each interval $Q$ in $\mathbb{T}$, inequality (3.1) implies that

$$
\underset{x \in Q}{\operatorname{ess} \sup _{0}} \omega(x) \leq R H_{\infty}(\omega) f_{Q} e^{\varphi(x)} d x \leq R H_{\infty}(\omega) A_{\infty}(\omega) e^{\varphi_{Q}} .
$$

Taking logarithms, we see that

$$
\underset{x \in Q}{\operatorname{ess} \sup } \varphi(x) \leq \varphi_{Q}+\log \left(R H_{\infty}(\omega) A_{\infty}(\omega)\right),
$$

and so inequality (3.4) holds with $C_{4} \leq \log \left(R H_{\infty}(\omega) A_{\infty}(\omega)\right)$. 
Conversely, if inequality (3.4) holds, then by Jensen's inequality

$$
\underset{x \in Q}{\operatorname{ess} \sup } \omega(x) \leq \exp \left\{C_{4}+\varphi_{Q}\right\} \leq e^{C_{4}} f_{Q} \exp \varphi(x) d x=e^{C_{4}} f_{Q} \omega .
$$

Thus $\omega$ satisfies the $R H_{\infty}$ property with constant $R H_{\infty}(\omega) \leq e^{C_{4}}$.

(e) We show that $C_{5} \leq R H_{p}(\omega) A_{\infty}(\omega)$ and $R H_{p}(\omega) \leq C_{5}^{1 / p}$, and that this bound on $C_{5}$ depends only on $p$ and on $R H_{p}(\omega)$. In terms of $\varphi$, the $R H_{p}$ expression for a given interval $Q$ is

$$
\left(f_{Q} \omega^{p}\right)^{1 / p}\left(f_{Q} \omega\right)^{-1}=\left(f_{Q} e^{p\left(\varphi(x)-\varphi_{Q}\right)} d x\right)^{1 / p}\left(f_{Q} e^{\varphi(x)-\varphi_{Q}} d x\right)^{-1} .
$$

Also, if $\omega$ is in $R H_{p}$, then $\omega$ is in $A_{\infty}$ and $A_{\infty}(\omega)$ depends only on $p$ and on $R H_{p}(\omega)$. It follows that

$$
\begin{aligned}
& \left(f_{Q} \exp \left\{p\left(\varphi(x)-\varphi_{Q}\right)\right\} d x\right)^{1 / p} \leq \\
& \quad \leq R H_{p}(\omega) f_{Q} \exp \left\{\varphi(x)-\varphi_{Q}\right\} d x \leq R H_{p}(\omega) A_{\infty}(\omega) .
\end{aligned}
$$

Thus inequality (3.5) holds with $C_{5} \leq R H_{p}(\omega) A_{\infty}(\omega)$, and this bound depends only on $p$ and on $R H_{p}(\omega)$.

By Jensen's inequality, $f_{Q} \exp \left\{\varphi(x)-\varphi_{Q}\right\} d x \geq 1$. Thus if inequality (3.5) holds, then

$$
\left(f_{Q} \exp \left\{p\left(\varphi(x)-\varphi_{Q}\right)\right\} d x\right)^{1 / p}\left(f_{Q} \exp \left\{\varphi(x)-\varphi_{Q}\right\} d x\right)^{-1} \leq C_{5}^{1 / p} .
$$

Thus $\omega$ is in $R H_{p}$ and $R H_{p}(\omega) \leq C_{5}^{1 / p}$.

The same arguments go through for the dyadic classes $A_{p}^{d}$ and $R H_{p}^{d}$.

Muckenhoupt's $A_{p}$ weights are closely related to functions of bounded mean oscillation.

Definition 4. A real-valued function $f \in L^{1}(\mathbb{T})$ lies in the space $\operatorname{BMO}(\mathbb{T})$ of functions of bounded mean oscillation on the circle if its BMO norm is finite:

$$
\|f\|_{*}:=\sup _{Q \subset \mathbb{T}} f_{Q}\left|f(x)-f_{Q}\right| d x<\infty .
$$

Dyadic $\mathrm{BMO}$ of the circle, written $\mathrm{BMO}_{d}(\mathbb{T})$, is the space of functions that satisfy the corresponding estimate where the supremum is taken over all dyadic subintervals $I \in \mathcal{D}$ of $[0,1]$. The dyadic BMO norm of $f$ is denoted by $\|f\|_{d}$. 
Elements of $\mathrm{BMO}$, or of $\mathrm{BMO}_{d}$, that differ only by an additive constant are equivalent; thus BMO and $\mathrm{BMO}_{d}$ are subspaces of $L_{\text {loc }}^{1} / \mathbb{R}$.

For $1 \leq p \leq \infty$, if $\omega$ is in $A_{p}$ then $\varphi:=\log \omega$ is in BMO, with BMO norm depending only on the $A_{p}$ constant $A_{p}(\omega)$. See for example [3, p. 409]. The same is true for $R H_{p}$ weights. Specifically, we have the following result; we omit the proof.

Lemma 2. Suppose $1 \leq p \leq \infty$. If $\omega$ is in $A_{p}$ then $\varphi:=\log \omega$ is in BMO. For $1<p<\infty$,

$$
\|\varphi\|_{*} \leq A_{p}(\omega)+(p-1) A_{p}(\omega)^{1 /(p-1)} .
$$

For $p=1,\|\varphi\|_{*} \leq 2 A_{1}(\omega)$. For $p=\infty,\|\varphi\|_{*}$ depends only on $A_{\infty}(\omega)$. For $1<p \leq \infty$, if $\omega$ is in $R H_{p}$ then $\varphi:=\log \omega$ is in $\mathrm{BMO}$, with $\|\varphi\|_{*}$ depending only on $R H_{p}(\omega)$ and on $p$. The analogous statements hold in the dyadic setting.

We use a characterization of the dyadic BMO functions on the circle in terms of the size of Haar coefficients. The Haar function $h_{I}$ associated with the dyadic interval $I$ is given by $h_{I}(x)=|I|^{-1 / 2}$ if $x$ is in the left half of $I$, $h_{I}(x)=-|I|^{-1 / 2}$ if $x$ is in the right half of $I$, and $h_{I}=0$ otherwise. The Haar coefficient over $I$ of $f$ is $\left(f, h_{I}\right):=\int_{I} f(x) h_{I}(x) d x$. The Haar series for $f$ is

$$
f(x):=\sum_{I \in \mathcal{D}}\left(f, h_{I}\right) h_{I}(x),
$$

and the $L^{2}$-norm of $f$ is given in terms of the Haar coefficients by

$$
\|f\|_{2}=\left[\sum_{J \in \mathcal{D}}\left(f, h_{J}\right)^{2}\right]^{1 / 2} .
$$

The John-Nirenberg Theorem [8] implies that for each $p \geq 1$ and for each $f$ in $L^{1}(\mathbb{T})$, the expression

$$
\|f\|_{d, p}:=\sup _{I \in \mathcal{D}}\left(f_{I}\left|f(x)-f_{I}\right|^{p} d x\right)^{1 / p}
$$

is comparable to the dyadic BMO norm $\|f\|_{d}$.

A function $f \in L^{1}(\mathbb{T})$ of mean value zero is in $\mathrm{BMO}_{d}(\mathbb{T})$ if and only if there is a constant $C$ such that for all $I \in \mathcal{D}$,

$$
\sum_{J \subset I, J \in \mathcal{D}}\left(f, h_{J}\right)^{2} \leq C|I| .
$$

The smallest such constant $C$ is equal to $\|f\|_{d, 2}^{2}$. Since the sum in inequality (3.7) ranges over only dyadic intervals $J$, there is no need to restrict the interval $I$ itself to be dyadic. 


\section{Proofs of Theorems 1 and 2}

We begin this section with three lemmas, which we then use to prove the geometric-arithmetic averaging result for both $A_{p}$ and $R H_{p}$.

Lemma 3 below gives an estimate on Haar expansions of $\mathrm{BMO}_{d}$ functions. Lemmas 4 and 5, which rely on the estimates (4.1) and (4.2) from Lemma 3, will allow us to pass from the dyadic versions to the non-dyadic versions of the inequalities that characterize $A_{p}$ and $R H_{p}$.

Throughout this section we use the following notation. Let $\mathcal{D}_{n}:=\{I \in$ $\left.\mathcal{D}|| I \mid=2^{-n}\right\}$ be the collection of dyadic intervals of length $2^{-n}$, for $n=0$, $1,2, \ldots$ Expanding each $\varphi^{t}$ in Haar series, we have

$$
\begin{aligned}
\varphi(x) & =\int_{0}^{1} \sum_{J \in \mathcal{D}}\left(\varphi^{t}, h_{J}\right) h_{J}(x+t) d t \\
& =\sum_{n=0}^{\infty} \int_{0}^{1} \sum_{J \in \mathcal{D}_{n}}\left(\varphi^{t}, h_{J}\right) h_{J}(x+t) d t=\sum_{n=0}^{\infty} \varphi_{n}(x),
\end{aligned}
$$

so that $\varphi_{n}$ is the translation-average over $t$ of the slices at scale $2^{-n}$ of the Haar expansions for the functions $\varphi^{t}$.

Fix an interval $Q \subset \mathbb{T}$; this $Q$ need not necessarily be dyadic. Split the sum for $\varphi(x)$, at the scale of $|Q|$, into two parts $\varphi_{A}$ and $\varphi_{B}$ in which the dyadic intervals $J$ are respectively small and large compared with $Q$ :

$$
\varphi=\varphi_{A}+\varphi_{B}, \quad \varphi_{A}(x):=\sum_{n: 2^{-n}<|Q|} \varphi_{n}(x), \quad \varphi_{B}(x):=\sum_{n: 2^{-n} \geq|Q|} \varphi_{n}(x) .
$$

The following result is proved in the course of the proof of Theorem 2 of [13].

Lemma 3. Suppose that $\left\{\varphi^{t}\right\}_{t \in[0,1]}$ is a family of dyadic BMO functions on $\mathbb{T}, \varphi^{t} \in \mathrm{BMO}_{d}(\mathbb{T})$, such that

(i) the mapping $t \mapsto \varphi^{t}$ is measurable,

(ii) the $\mathrm{BMO}_{d}$ constants $\left\|\varphi^{t}\right\|_{d}$ are uniformly bounded, independent of $t \in[0,1]$, and

(iii) for each $t \in[0,1]$, the function $\varphi^{t}$ has mean value zero on $\mathbb{T}$.

Then there are constants $C_{A}$ and $C_{B}$ depending on the bound on the $\mathrm{BMO}_{d}$ constants $\left\|\varphi^{t}\right\|_{d}$, and independent of $Q$, such that for each interval $Q \subset \mathbb{T}$ and for each point $x_{0} \in Q$,

$$
\begin{aligned}
& \frac{1}{|Q|} \int_{Q}\left|\varphi_{A}(x)\right|^{2} d x \leq C_{A}, \\
& \frac{1}{|Q|} \int_{Q}\left|\varphi_{B}(x)-\varphi_{B}\left(x_{0}\right)\right| d x \leq C_{B} .
\end{aligned}
$$


Lemma 4. Let $\beta$ be a real number. Suppose $\left\{\omega^{t}\right\}_{t \in[0,1]}$ is a family of nonnegative locally integrable functions on $\mathbb{T}$ such that for all $\varphi^{t}:=\log \omega^{t}$, hypotheses $(i)-($ iii $)$ of Lemma 3 hold. Let $\varphi(x)=\log \Omega(x):=\int_{0}^{1} \log \omega^{t}(x+t) d t$. Suppose there is a constant $C^{d}(\beta)$ such that for all $t \in[0,1]$ and for all dyadic intervals $I \subset \mathbb{T}$,

$$
f_{I} \exp \left[\beta\left(\varphi^{t}(x)-\varphi_{I}^{t}\right)\right] d x \leq C^{d}(\beta) .
$$

Then there is a constant $C(\beta)$, depending only on $C^{d}(\beta)$, such that for all intervals $Q \subset \mathbb{T}$,

$$
f_{Q} \exp \left[\beta\left(\varphi(x)-\varphi_{Q}\right)\right] d x \leq C(\beta) .
$$

In fact, for many choices of $\beta$, hypothesis (ii) of Lemma 3 is implied by inequality (4.3), together with Lemmas 1 and 2. This point is made clear in the proof of Theorems 1 and 2 .

Proof. We first establish an inequality that controls the exponentials of the Haar expansions of the $\varphi^{t}$. By inequality (4.3), for each dyadic interval $I \subset \mathbb{T}$ we have

$$
\begin{aligned}
& f_{I} \exp \left[\beta \sum_{J \subset I, J \in \mathcal{D}}\left(\varphi^{t}, h_{J}\right) h_{J}(x)\right] d x= \\
&=f_{I} \exp \left[\beta \sum_{J \in \mathcal{D}}\left(\varphi^{t}, h_{J}\right) h_{J}(x)-\beta \sum_{J \supseteq I, J \in \mathcal{D}}\left(\varphi^{t}, h_{J}\right) h_{J}(x)\right. \\
&\left.-\beta \sum_{J \cap I=\emptyset, J \in \mathcal{D}}\left(\varphi^{t}, h_{J}\right) h_{J}(x)\right] d x \\
&= f_{I} \exp \left[\beta\left(\varphi^{t}(x)-\varphi_{I}^{t}\right)\right] d x \leq C^{d}(\beta) .
\end{aligned}
$$

We have used the fact that the average $f_{I}$ of a function $f \in L^{1}(\mathbb{T})$ over an interval $I$ containing the point $x$ can be written as

$$
f_{I}=f_{I} \sum_{J \in \mathcal{D}}\left(f, h_{J}\right) h_{J}(s) d s=\sum_{J \supsetneq I, J \in \mathcal{D}}\left(f, h_{J}\right) h_{J}(x),
$$

and the observation that $\exp \left[-\beta \sum_{J \cap I=\emptyset, J \in \mathcal{D}}\left(\varphi^{t}, h_{J}\right) h_{J}(x)\right]=1$ for $x \in I$.

Fix an interval $Q \subset \mathbb{T}$, not necessarily dyadic. For each $x \in Q$ we have

$$
\begin{aligned}
\left|\varphi_{B}(x)-f_{Q} \varphi(s) d s\right| & \leq f_{Q}\left|\varphi_{B}(x)-\varphi(s)\right| d s \\
& \leq f_{Q}\left|\varphi_{A}(s)\right| d s+f_{Q}\left|\varphi_{B}(x)-\varphi_{B}(s)\right| d s \leq \sqrt{C_{A}}+C_{B},
\end{aligned}
$$

by Cauchy-Schwarz and the estimates (4.1) and (4.2) from Lemma 3. 
Therefore

$$
\begin{aligned}
f_{Q} \exp \left[\beta\left(\varphi(x)-\varphi_{Q}\right)\right] d x & =f_{Q} \exp \left[\beta \varphi_{A}(x)\right] \exp \left[\beta\left(\varphi_{B}(x)-\varphi_{Q}\right)\right] d x \\
& \leq \exp \left[\beta\left(\sqrt{C_{A}}+C_{B}\right)\right] f_{Q} \exp \left[\beta \varphi_{A}(x)\right] d x
\end{aligned}
$$

Thus it suffices to bound the quantity

$$
\begin{aligned}
f_{Q} \exp \left[\beta \varphi_{A}(x)\right] d x & =f_{Q} \exp \left[\beta \sum_{2^{-n}<|Q|} \int_{0}^{1} \sum_{J \in \mathcal{D}_{n}}\left(\varphi^{t}, h_{J}\right) h_{J}(x+t) d t\right] d x \\
& =f_{Q} \exp \left[\int_{0}^{1} \beta \sum_{|J|<|Q|, J \in \mathcal{D}}\left(\varphi^{t}, h_{J}\right) h_{J}(x+t) d t\right] d x \\
& \leq \int_{0}^{1} f_{Q} \exp \left[\beta \sum_{|J|<|Q|, J \in \mathcal{D}}\left(\varphi^{t}, h_{J}\right) h_{J}(x+t)\right] d x d t \\
& =\int_{0}^{1} f_{Q+t} \exp \left[\beta \sum_{|J|<|Q|, J \in \mathcal{D}}\left(\varphi^{t}, h_{J}\right) h_{J}(x)\right] d x d t
\end{aligned}
$$

We have used Jensen's inequality and Tonelli's Theorem.

In order to apply inequality (4.5), we want to replace the interval $Q$ in the preceding expression by appropriate dyadic intervals. Fix $t$. There are two adjacent dyadic intervals $K_{t}$ and $L_{t}$ such that $Q+t \subset K_{t} \cup L_{t}$ and $|Q| \leq\left|K_{t}\right|=\left|L_{t}\right|<2|Q|$. Then

$$
\begin{aligned}
f_{Q} \exp \left[\beta \varphi_{A}(x)\right] d x & \leq \int_{0}^{1} \frac{1}{|Q|} \int_{Q+t} \exp \left[\beta \sum_{|J|<|Q|, J \in \mathcal{D}}\left(\varphi^{t}, h_{J}\right) h_{J}(x)\right] d x d t \\
\leq & \int_{0}^{1} \frac{2}{\left|K_{t}\right|} \int_{K_{t} \cup L_{t}} \exp \left[\beta \sum_{|J|<\left|K_{t}\right|, J \in \mathcal{D}}\left(\varphi^{t}, h_{J}\right) h_{J}(x)\right] d x d t \\
= & 2 \int_{0}^{1}\left\{\frac{1}{\left|K_{t}\right|} \int_{K_{t}} \exp \left[\beta \sum_{|J|<\left|K_{t}\right|, J \in \mathcal{D}}\left(\varphi^{t}, h_{J}\right) h_{J}(x)\right] d x\right. \\
& \left.+\frac{1}{\left|L_{t}\right|} \int_{L_{t}} \exp \left[\beta \sum_{|J|<\left|L_{t}\right|, J \in \mathcal{D}}\left(\varphi^{t}, h_{J}\right) h_{J}(x)\right] d x\right\} d t \\
\leq & 2 \int_{0}^{1} C^{d}(\beta)+C^{d}(\beta) d t=4 C^{d}(\beta),
\end{aligned}
$$

by inequality (4.5). Thus

$$
f_{Q} \exp \left[\beta\left(\varphi(x)-\varphi_{Q}\right)\right] d x \leq 4 C^{d}(\beta) \exp \left[\beta\left(\sqrt{C_{A}}+C_{B}\right)\right] .
$$


Taking the supremum over all intervals $Q \subset \mathbb{T}$, we see that inequality (4.4) holds for $\varphi=\log \Omega$, with constant $C(\beta)=4 C^{d}(\beta) \exp \left[\beta\left(\sqrt{C_{A}}+C_{B}\right)\right]$.

Lemma 5. Suppose $\left\{\omega^{t}\right\}_{t \in[0,1]}$ is a family of nonnegative locally integrable functions on $\mathbb{T}$ such that for all $\varphi^{t}:=\log \omega^{t}$, hypotheses $(i)$ and (iii) of Lemma 3 hold. As before, let $\varphi(x)=\log \Omega(x):=\int_{0}^{1} \log \omega^{t}(x+t) d t$.

(a) Suppose there is a constant $C_{3}^{d}$ such that for all $t \in[0,1]$ and for all dyadic intervals $I \subset \mathbb{T}$,

$$
\left[\varphi_{I}^{t}-\operatorname{ess} \inf _{x \in I} \varphi^{t}(x)\right] \leq C_{3}^{d} .
$$

Then there is a constant $C_{3}$ depending only on $C_{3}^{d}$ such that for all intervals $Q \subset \mathbb{T}$,

$$
\left[\varphi_{Q}-\underset{x \in Q}{\operatorname{ess} \inf } \varphi(x)\right] \leq C_{3}
$$

(b) Similarly, if there is a constant $C_{4}^{d}$ such that for all $t \in[0,1]$ and for all dyadic intervals $I \subset \mathbb{T}$,

$$
\left[\operatorname{ess}_{x \in I} \sup ^{t}(x)-\varphi_{I}^{t}\right] \leq C_{4}^{d},
$$

then there is a constant $C_{4}$ depending only on $C_{4}^{d}$ such that for all intervals $Q \subset \mathbb{T}$,

$$
\left[\underset{x \in Q}{\operatorname{ess} \sup } \varphi(x)-\varphi_{Q}\right] \leq C_{4} .
$$

Proof. Observe that hypothesis (ii) of Lemma 3 follows from the assumption in part $(a)$ or the assumption in part $(b)$, together with the dyadic versions of Lemmas 1 and 2. In particular, the $\mathrm{BMO}_{d}$ constants $\left\|\varphi^{t}\right\|_{d}$ depend only on $C_{3}^{d}$ or $C_{4}^{d}$.

(a) For each dyadic interval $I \subset \mathbb{T}$ and for a.e. $x \in I$, we have

$$
C_{3}^{d} \geq \varphi_{I}^{t}-\varphi^{t}(x)=-\sum_{J \subset I, J \in \mathcal{D}}\left(\varphi^{t}, h_{J}\right) h_{J}(x),
$$

using the observation that $\sum_{J \cap I=\emptyset, J \in \mathcal{D}}\left(\varphi^{t}, h_{J}\right) h_{J}(x)=0$ for $x \in I$.

Fix an interval $Q \subset \mathbb{T}$, not necessarily dyadic. For $x \in Q$ consider the quantity

$$
\begin{aligned}
\varphi_{Q}-\varphi(x) & =f_{Q}\left[\varphi_{A}(s)+\varphi_{B}(s)\right] d s-\left[\varphi_{A}(x)+\varphi_{B}(x)\right] \\
& =\underbrace{f_{Q} \varphi_{A}(s) d s}_{\text {(I) }}+\underbrace{\left[f_{Q} \varphi_{B}(s) d s-\varphi_{B}(x)\right]}_{\text {(II) }}+\underbrace{\left[-\varphi_{A}(x)\right]}_{\text {(III) }} .
\end{aligned}
$$


We bound the terms (I), (II), and (III) separately. By Lemma 3,

$$
(\mathrm{I}) \leq\left[f_{Q}\left|\varphi_{A}(s)\right|^{2} d s\right]^{1 / 2} \leq \sqrt{C_{A}} \text { and } \quad(\mathrm{II}) \leq f_{Q}\left|\varphi_{B}(s)-\varphi_{B}(x)\right| d s \leq C_{B}
$$

for all $x \in Q$. Also, given $x \in Q$ and $t \in[0,1]$, there is a unique dyadic interval $I_{x, t}$ such that $x+t \in I_{x, t}$ and $|Q| / 2 \leq\left|I_{x, t}\right|<|Q|$. Then $h_{J}(x+t)=0$ if $J \not \subset I_{x, t}$. It follows that

$$
\begin{aligned}
-\varphi_{A}(x) & =-\sum_{n: 2^{-n}<|Q|} \int_{0}^{1} \sum_{J \in \mathcal{D}_{n}}\left(\varphi^{t}, h_{J}\right) h_{J}(x+t) d t \\
& =-\int_{0}^{1} \sum_{|J|<|Q|, J \in \mathcal{D}}\left(\varphi^{t}, h_{J}\right) h_{J}(x+t) d t \\
& =-\int_{0}^{1} \sum_{J \subset I_{x, t}, J \in \mathcal{D}}\left(\varphi^{t}, h_{J}\right) h_{J}(x+t) d t \leq C_{3}^{d}
\end{aligned}
$$

for a.e. $x \in Q$, by inequality (4.6). So

$$
\varphi_{Q}-\underset{x \in Q}{\operatorname{ess} \inf _{0}} \varphi(x) \leq \sqrt{C_{A}}+C_{B}+C_{3}^{d}
$$

Thus inequality (3.3) holds with constant $C_{3}=\sqrt{C_{A}}+C_{B}+C_{3}^{d}$.

(b) In the case of $R H_{\infty}$, the same argument shows that inequality (3.4) holds with constant $C_{4}=C_{4}^{d}+C_{B}+\sqrt{C_{A}}$.

Proof of Theorems 1 and 2. We prove Theorems 1 and 2 together. Let $K$ denote any one of the classes $A_{p}$ with $1 \leq p \leq \infty$, or $R H_{p}$ with $1<p \leq \infty$. For $\omega$ in $K$ let $K(\omega)$ denote the corresponding $A_{p}$ constant or $R H_{p}$ constant. Let $K^{d}$ denote the corresponding dyadic class, and for $\omega^{t}$ in $K^{d}$ let $K^{d}\left(\omega^{t}\right)$ denote the corresponding constant.

It follows from the definitions of $A_{p}$ and $R H_{p}$ that if $\omega$ is in $K$ then for each constant $\lambda>0$ the weight $\lambda \omega$ is also in $K$, and $K(\lambda \omega)=K(\omega)$.

For each $t \in[0,1]$ let $\omega^{t}$ be a weight in $K^{d}$, and let $\varphi^{t}:=\log \omega^{t}$. Let $\varphi:=\log \Omega$. By hypothesis (iii) of Theorem 1 or $\left(i i i^{\prime}\right)$ of Theorem 2, the constants $K^{d}\left(\omega^{t}\right)$ are bounded independently of $t \in[0,1]$.

Without loss of generality, we may assume that for a.e. $t \in[0,1]$ the functions $\varphi^{t}$ have mean value zero. To see this, note that the mean value $\varphi_{\mathbb{T}}^{t}:=f_{\mathbb{T}} \varphi^{t}(x) d x$ is finite for a.e. $t$ by hypothesis $(i i)$. Let $\widetilde{\varphi}^{t}:=\varphi^{t}-\varphi_{\mathbb{T}}^{t}$. Then for $x \in \mathbb{T}$,

$$
\begin{aligned}
\Omega(x) & =\exp \left\{\int_{0}^{1} \varphi^{t}(x+t) d t\right\}=\exp \left\{\int_{0}^{1} \varphi_{\mathbb{T}}^{t} d t\right\} \exp \left\{\int_{0}^{1} \widetilde{\varphi}^{t}(x+t) d t\right\} \\
& =\exp \left\{\int_{0}^{1} \int_{0}^{1} \log \omega^{t}(x) d x d t\right\} \exp \left\{\int_{0}^{1} \widetilde{\varphi}^{t}(x+t) d t\right\} .
\end{aligned}
$$


Denote the second term on the right-hand side by $\widetilde{\Omega}(x)$. The first term on the right-hand side is finite by hypothesis $(i i)$ of Theorems 1 and 2 , and is positive. Thus $\Omega$ is in $K$ if and only if $\widetilde{\Omega}$ is in $K$. Moreover, $K(\Omega)=K(\widetilde{\Omega})$.

We show that hypotheses $(i)-($ iii $)$ of Lemma 3 hold here. The mapping $t \mapsto \widetilde{\varphi}^{t}=\left(\log \omega^{t}\right)-\varphi_{\mathbb{T}}^{t}$ is measurable, since $t \mapsto \omega^{t}$ is measurable by hypothesis. By the dyadic version of Lemma 2, the $\mathrm{BMO}_{d}$ norms $\left\|\widetilde{\varphi}^{t}\right\|_{d}=\left\|\varphi^{t}\right\|_{d}$ are uniformly bounded, by a constant depending only on $p$ and on the bound on the $K^{d}$ constants of the dyadic weights $\omega^{t}$. Each $\widetilde{\varphi}^{t}$ has mean value zero, by construction.

For convenience we now drop the tildes, writing $\varphi^{t}$ for $\widetilde{\varphi}^{t}$ and $\Omega$ for $\widetilde{\Omega}$ from here on.

As an aside, we note that $\log \Omega$ is in BMO (see [5]), and so by the JohnNirenberg Theorem the function $\Omega^{\delta}$ is in $A_{p}$ for $\delta>0$ sufficiently small. We now prove that $\Omega$ itself is in $A_{p}$.

Case 1: $K=A_{\infty}$. By the dyadic version of Lemma 1(a), there is a constant $C_{1}^{d}$ depending only on the bound on the $A_{\infty}^{d}$ constants of the $\omega^{t}$ such that each $\varphi^{t}$ satisfies inequality (4.3) with $\beta=1$ :

$$
f_{I} \exp \left\{\varphi^{t}(x)-\varphi_{I}^{t}\right\} d x \leq C_{1}^{d}
$$

Take $C^{d}(1)=C_{1}^{d}$. Then by Lemma $4, \varphi=\log \Omega$ satisfies inequality (4.4) with $\beta=1$ : there is a constant $C(1)$ depending only on $C^{d}(1)$ such that

$$
f_{Q} \exp \left\{\varphi(x)-\varphi_{Q}\right\} d x \leq C(1)
$$

Take $C_{1}=C(1)$. Lemma $1(a)$ now implies that $\Omega \in A_{\infty}$, with $A_{\infty}$ constant bounded by $C_{1}$. The dependence of the constants is illustrated in the upper row of Figure 1 (taking $p=\infty$ there). We see that $A_{\infty}(\Omega)$ depends only on the bound on the $A_{\infty}^{d}$ constants of the weights $\omega^{t}$.

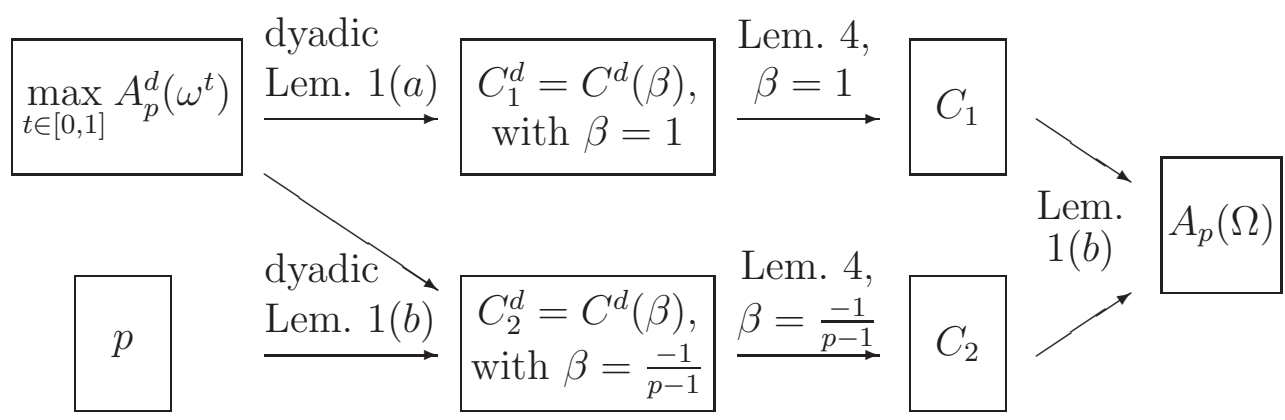

Figure 1: Dependence of the constants in the proof of Theorem 1, for the case $K=A_{p}$ with $1<p<\infty$. 
Case 2: $K=A_{p}, 1<p<\infty$. As for case 1, using Lemma 1(b) and Lemma 4 with both $\beta=1$ and $\beta=-1 /(p-1)$. Figure 1 illustrates the dependence of the constants. We find that $A_{p}(\Omega)$ depends only on $p$ and on the bound on the $A_{p}^{d}$ constants of the $\omega^{t}$.

Case 3: $K=A_{1}$. As for case 1, using Lemma 1(c), Lemma 4 with $\beta=1$, and Lemma 5. The constant $A_{1}(\Omega)$ depends only on the bound on the $A_{1}^{d}$ constants of the $\omega^{t}$.

Case 4: $K=R H_{\infty}$. As for case 1, using Lemma 1(d) and Lemma 5. The constant $R H_{\infty}(\Omega)$ depends only on the bound on the $R H_{\infty}^{d}$ constants of the $\omega^{t}$.

Case 5: $K=R H_{p}$. As for case 1, using Lemma 1(e) and Lemma 4 with $\beta=p$. We find that $R H_{p}(\Omega)$ depends only on $p$ and on the bound on the $R H_{p}^{d}$ constants of the $\omega^{t}$.

This completes the proof of Theorems 1 and 2 .

Remark. An alternative proof of Theorem 1 for $K=A_{p}$, with $1<p \leq \infty$, can be obtained as follows from the result for $K=A_{1}$, using factorization of $A_{p}$ weights [10]. Suppose $1 \leq p<\infty$. If $\omega_{1}$ and $\omega_{2}$ are $A_{1}$ weights, then $\omega=\omega_{1} \omega_{2}^{1-p}$ is an $A_{p}$ weight, with constant $A_{p}(\omega) \leq A_{1}\left(\omega_{1}\right) A_{1}\left(\omega_{2}\right)^{p-1}$. Conversely, if $\omega \in A_{p}$, then there exist $\omega_{1}$ and $\omega_{2}$ in $A_{1}$ such that $\omega=\omega_{1} \omega_{2}^{1-p}$. The $A_{1}$ constants of $\omega_{1}$ and $\omega_{2}$ depend only on $p$ and on the $A_{p}$ constants of $\omega$, as noted in $\left[6\right.$, p. 717]. The analogous results hold in the dyadic setting $A_{p}^{d}$.

Lemma 6. If Theorem 1 holds for $A_{1}$, then it holds for each $A_{p}, 1 \leq p \leq \infty$.

Proof. By the (dyadic) factorization theorem, for each $t \in[0,1]$ there exist $\omega_{1}^{t}, \omega_{2}^{t} \in A_{1}^{d}$ such that $\omega^{t}=\omega_{1}^{t}\left(\omega_{2}^{t}\right)^{1-p}$. Furthermore $A_{1}\left(\omega_{1}^{t}\right)$ and $A_{1}\left(\omega_{2}^{t}\right)$ are uniformly bounded, independent of $t \in[0,1]$, by a constant depending on $p$ and on the bound for the constants $A_{p}\left(\omega^{t}\right)$. Then

$$
\begin{aligned}
\Omega(x) & =\exp \left\{\int_{0}^{1} \log \omega^{t}(x+t) d t\right\} \\
& =\exp \left\{\int_{0}^{1} \log \left[\omega_{1}^{t}(x+t)\left(\omega_{1}^{t}(x+t)\right)^{1-p}\right] d t\right\} \\
& =\exp \left\{\int_{0}^{1} \log \omega_{1}^{t}(x+t) d t+(1-p) \int_{0}^{1}\left(\omega_{1}^{t}(x+t)\right) d t\right\} \\
& =\left[\exp \left\{\int_{0}^{1} \log \omega_{1}^{t}(x+t) d t\right\}\right]\left[\exp \left\{\int_{0}^{1} \log \omega_{2}^{t}(x+t) d t\right\}\right]^{1-p} .
\end{aligned}
$$

By hypothesis, both of the expressions in square brackets are in $A_{1}$. Therefore $\Omega$ is in $A_{p}$ as required. Furthermore, the $A_{p}$ constant of $\Omega$ depends only on the $A_{p}^{d}$ constants of the weights $\omega^{t}$. 
The result for $A_{\infty}$ follows immediately from the $A_{p}$ case, $1 \leq p<\infty$, using the observations on the dependence of the constants made before Lemma 1 above.

\section{The translation-average of doubling weights}

It appears that the obstacle to the translation-average $\omega(x)=\int_{0}^{1} \omega^{t}(x+t) d t$ of $A_{p}^{d}$ weights $\omega^{t}$ being in $A_{p}$ is that the assumption of the $\omega^{t}$ being dyadic doubling is insufficient to guarantee that the translation-average is actually doubling [16]. A natural conjecture is that if the translation-average of a given family of $A_{p}^{d}$ weights is in fact doubling, then this doubling weight also belongs to true $A_{p}$. As a step in this direction, we show that the presumably stronger assumption that each of the $A_{p}^{d}$ weights $\omega^{t}$ is doubling (in other words, that $\omega^{t}$ is in $A_{p}$ ) does imply that their translation-average $\omega$ is in $A_{p}$.

Theorem 3. Suppose $\left\{\omega^{t}\right\}_{t \in[0,1]}$ is a family of doubling weights on $\mathbb{T}$, with doubling constants bounded by a constant $C_{\mathrm{db}}$ independent of $t \in[0,1]$. Suppose the mapping $t \mapsto \omega^{t}$ is measurable. Fix $p$ with $1 \leq p \leq \infty$, and suppose each $\omega^{t}$ is in $A_{p}^{d}$, with $A_{p}^{d}$ constant bounded by a constant $V_{p, d}$ independent of $t \in[0,1]$. Then the translation-average

$$
\omega(x):=\int_{0}^{1} \omega^{t}(x+t) d t
$$

belongs to $A_{p}$ on $\mathbb{T}$, with $A_{p}$ constant depending only on $p, V_{p, d}$, and $C_{\mathrm{dbl}}$.

Similarly, for $p$ with $1<p \leq \infty$, if each $\omega^{t}$ is in $R H_{p}^{d}$, with the supremum in the defining inequality for $R H_{p}^{d}$ (Definition 3) bounded by a constant $V_{p, d}$ independent of $t \in[0,1]$, then the translation-average $\omega(x)$ belongs to $R H_{p}$ on $\mathbb{T}$, with $R H_{p}(\omega)$ depending on $p, V_{p, d}$, and $C_{\mathrm{dbl}}$.

Proof. A doubling weight assigns comparable mass, with a constant depending only on the doubling constant, to any given interval $Q$ and to each of the dyadic intervals at scale $|Q|$ that intersect $Q$. (This observation can fail if the weight is dyadic doubling but not doubling.)

As a consequence, for fixed $p$ with $1 \leq p \leq \infty$, an $A_{p}^{d}$ weight is doubling if and only if it is actually in $A_{p}$. Moreover the $A_{p}$ constant depends only on the $A_{p}^{d}$ constant and the doubling constant, which in turn depend only on the $A_{p}$ constant. Similarly, for fixed $p$ with $1<p \leq \infty$, an $R H_{p}^{d}$ weight is doubling if and only if it is actually in $R H_{p}$. For example, one finds that for a doubling $A_{p}^{d}$ weight $\omega^{t}$ with $1<p<\infty$, the $A_{p}$ constant of $\omega^{t}$ is bounded by $V_{p}:=2^{2 p-1} V_{p, d} C_{\mathrm{dbl}}^{2}$, while for a doubling $R H_{p}^{d}$ weight $\omega^{t}$ with $1<p<\infty$, the $R H_{p}$ constant of $\omega^{t}$ is bounded by $V_{p}:=2^{2 / p} V_{p, d} C_{\mathrm{dbl}}^{2}$. 
Theorem 3 now follows easily for $A_{p}, 1<p<\infty$, using Muckenhoupt's original identification of $A_{p}$ in terms of the boundedness of the HardyLittlewood maximal function $M$, defined by $M f(x):=\sup _{Q \ni x} f_{Q}|f(y)| d y$. In particular, for these $p$, a nonnegative locally integrable function $\omega$ is in $A_{p}$ if and only if there is a constant $C$ such that for all locally integrable functions $f$,

$$
\int_{\mathbb{R}}|M f(x)|^{p} \omega(x) d x \leq C \int_{\mathbb{R}}|f(x)|^{p} \omega(x) d x .
$$

Moreover, if inequality (5.1) holds then $\omega \in A_{p}$ and $A_{p}(\omega) \leq C$, while if $\omega \in A_{p}$ then inequality (5.1) holds with $C$ depending on $p$ and $A_{p}(\omega)$.

For $R H_{p}, 1<p<\infty$, Theorem 3 follows from Minkowski's Integral Inequality and the observation above on comparable mass. The cases of $A_{1}$, $A_{\infty}$, and $R H_{\infty}$ are also straightforward, and we omit the proofs.

\section{Generalizations to the polydisc}

We extend the above results for $A_{p}(\mathbb{T})$ and $R H_{p}(\mathbb{T})$ to the setting of the polydisc. For ease of notation, the statements and proofs given below are expressed for the bidisc. However, they generalize immediately to the polydisc with arbitrarily many factors.

The theory of product weights was developed by K.-C. Lin in his thesis [11], while the dyadic theory was developed in Buckley's paper [1]. The product $A_{p}$ and $R H_{p}$ weights and the product doubling weights, and their dyadic analogues, are defined exactly as in Definitions 1-3 in Section 3, with intervals in $\mathbb{T}$ being replaced by rectangles in $\mathbb{T} \otimes \mathbb{T}$. It follows that a product weight belongs to $A_{p}(\mathbb{T} \otimes \mathbb{T})$ if and only if it belongs to $A_{p}(\mathbb{T})$ in each variable separately.

To be precise, $\omega \in A_{p}(\mathbb{T} \otimes \mathbb{T})$ if and only if $\omega(\cdot, y) \in A_{p}(\mathbb{T})$ uniformly for a.e. $y \in \mathbb{T}$ and $\omega(x, \cdot) \in A_{p}(\mathbb{T})$ uniformly for a.e. $x \in \mathbb{T}$. In one direction this is a consequence of the Lebesgue Differentiation Theorem, letting one side of the rectangle shrink to a point. The converse uses the equivalence between $\omega \in A_{p}(\mathbb{T} \otimes \mathbb{T})$ and inequality (5.1) with $M$ replaced by the strong maximal function $\left[14\right.$, p.83]. Further, the $A_{p}(\mathbb{T} \otimes \mathbb{T})$ constant depends only on the two $A_{p}(\mathbb{T})$ constants, and vice versa. The analogous characterizations in terms of the separate variables hold for product $R H_{p}$ weights and for product doubling weights, and for the dyadic product $A_{p}, R H_{p}$, and doubling weights.

Theorem 4. Fix $p$ with $1 \leq p \leq \infty$. Let $\left\{\omega^{s, t}\right\}_{s, t \in[0,1]}$ be a family of dyadic $A_{p}$ weights on the boundary of the bidisc, $\omega^{s, t} \in A_{p}^{d}(\mathbb{T} \otimes \mathbb{T})$, such that

(i) the mapping $(s, t) \mapsto \omega^{s, t}$ is measurable, 
(ii) an appropriate average of the logarithms of the weights $\omega^{s, t}$ is finite:

$$
\int_{0}^{1} \int_{0}^{1} \int_{0}^{1} \int_{0}^{1}\left|\log \omega^{s, t}(x, y)\right| d x d y d s d t<\infty
$$

and

(iii) the $A_{p}^{d}(\mathbb{T} \otimes \mathbb{T})$ constants $A_{p}^{d}\left(\omega^{s, t}\right)$ are uniformly bounded, independent of $s, t \in[0,1]$.

Then the product geometric-arithmetic average

$$
\Omega(x, y):=\exp \left\{\int_{0}^{1} \int_{0}^{1} \log \omega^{s, t}(x+s, y+t) d s d t\right\}
$$

of the dyadic weights $\omega^{s, t}$ belongs to $A_{p}(\mathbb{T} \otimes \mathbb{T})$, with $A_{p}$ constant depending on $p$ and on the bound on the $A_{p}^{d}$ constants of the weights $\omega^{s, t}$.

Similarly, for fixed $p$ with $1<p \leq \infty$, if each $\omega^{s, t}$ is in $R H_{p}^{d}(\mathbb{T} \otimes \mathbb{T})$, if hypotheses (i) and (ii) hold, and if

$\left(i i i^{\prime}\right)$ the $R H_{p}^{d}(\mathbb{T} \otimes \mathbb{T})$ constants $R H_{p}^{d}\left(\omega^{s, t}\right)$ are uniformly bounded, independent of $s, t \in[0,1]$,

then $\Omega(x, y)$ lies in $R H_{p}(\mathbb{T} \otimes \mathbb{T})$, with $R H_{p}$ constant depending on $p$ and on the bound on the $R H_{p}^{d}$ constants of the $\omega^{s, t}$.

Proof. The proof is by iteration of the one-parameter argument, relying on Lemma 1 and Theorems 1 and 2. We give the argument for $K=A_{p}$, $1<p<\infty$. The other cases follow from similar iteration arguments; we omit the proofs.

We show that, for a.e. fixed $y, \Omega(x, y)$ belongs to $A_{p}(\mathbb{T})$ in the variable $x$, with $A_{p}$ constant independent of $y$. The hypotheses of Theorem 1 follow immediately from our assumptions. In particular, $s \mapsto \omega^{s, t}(\cdot, y)$ is measurable for each $t$ and a.e. $y$, and $\omega^{s, t}(\cdot, y)$ belongs to $A_{p}^{d}(\mathbb{T})$ in the first variable for all $s, t$ and for a.e. $y$, with constants independent of $s, t$, and $y$. Fix such a $y$; for emphasis we'll denote it by $y^{*}$.

Applying Theorem 1, we see that the function

$$
\Omega_{1}\left(x, y^{*}\right):=\exp \left\{\int_{0}^{1} \log \omega^{s, t}\left(x+s, y^{*}\right) d s\right\}
$$

belongs to $A_{p}(\mathbb{T})$ in $x$, with constant independent of $y^{*}$. Let

$$
\varphi_{1}\left(x, y^{*}\right):=\log \Omega_{1}\left(x, y^{*}\right)=\int_{0}^{1} \log \omega^{s, t}\left(x+s, y^{*}\right) d s .
$$


By Lemma 1(b) there are constants $C_{1}$ and $C_{2}$, depending only on $p$ and on the $A_{p}^{d}(\mathbb{T} \otimes \mathbb{T})$ constants of the $\omega^{s, t}$, such that

$$
\begin{aligned}
& \sup _{Q} f_{Q} \exp \left\{\varphi_{1}\left(x, y^{*}\right)-\left(\varphi_{1}\left(\cdot, y^{*}\right)\right)_{Q}\right\} d x \leq C_{1} \\
& \sup _{Q} f_{Q} \exp \left\{\frac{-1}{p-1}\left[\varphi_{1}\left(x, y^{*}\right)-\left(\varphi_{1}\left(\cdot, y^{*}\right)\right)_{Q}\right]\right\} d x \leq C_{2} .
\end{aligned}
$$

We show that the same inequalities hold when $\varphi_{1}$ is replaced by

$$
\varphi\left(x, y^{*}\right):=\log \Omega\left(x, y^{*}\right)=\int_{0}^{1} \int_{0}^{1} \log \omega^{s, t}\left(x+s, y^{*}+t\right) d s d t .
$$

Fix an interval $Q$. An application of Fubini's Theorem shows that

$$
\begin{aligned}
\varphi\left(x, y^{*}\right)-\left(\varphi\left(\cdot, y^{*}\right)\right)_{Q}= \\
=\int_{0}^{1} \int_{0}^{1} \varphi^{s, t}\left(x+s, y^{*}+t\right) d s d t-\int_{Q} \int_{0}^{1} \int_{0}^{1} \varphi^{s, t}\left(x^{\prime}+s, y^{*}+t\right) d s d t d x^{\prime} \\
=\int_{0}^{1}\left[\varphi_{1}\left(x, y^{*}+t\right)-\left(\varphi_{1}\left(\cdot, y^{*}+t\right)\right)_{Q}\right] d t .
\end{aligned}
$$

Then by Jensen's inequality and Tonelli's Theorem,

$$
\begin{aligned}
f_{Q} \exp \{ & \left.\varphi\left(x, y^{*}\right)-\left(\varphi\left(\cdot, y^{*}\right)\right)_{Q}\right\} d x= \\
\leq & f_{Q} \int_{0}^{1} \exp \left\{\varphi_{1}\left(x, y^{*}+t\right)-\left(\varphi_{1}\left(\cdot, y^{*}+t\right)\right)_{Q}\right\} d t d x \\
& =\int_{0}^{1} f_{Q} \exp \left\{\varphi_{1}\left(x, y^{*}+t\right)-\left(\varphi_{1}\left(\cdot, y^{*}+t\right)\right)_{Q}\right\} d x d t \leq C_{1}
\end{aligned}
$$

using inequality (6.1) with $y^{*}$ replaced by $y^{*}+t$ for a.e. $t$.

The same argument shows that inequality (6.2) holds for $\varphi\left(\cdot, y^{*}\right)$ for a.e. $y^{*}$. Lemma 1 then implies that $\Omega\left(x, y^{*}\right)$ belongs to $A_{p}(\mathbb{T})$ in $x$ for a.e. $y^{*}$, with uniform constants.

In an identical fashion, we find that $\Omega\left(x^{*}, y\right)$ belongs to $A_{p}(\mathbb{T})$ in $y$ for a.e. $x^{*}$, with uniform constants, which proves the theorem for $K=A_{p}$, $1<p<\infty$.

Remark. As in the one-parameter case, there is an alternative proof of the geometric-arithmetic averaging result (Theorem 4 ) for $A_{p}(\mathbb{T} \otimes \mathbb{T})$ where $1<p \leq \infty$, relying on the $A_{1}(\mathbb{T} \otimes \mathbb{T})$ case and the generalization to the bidisc setting [7] of the $A_{p}$ factorization theorem. Moreover, the product $A_{p}$ result can also be derived from the one-parameter result using the maximal function characterization of this weight class. 
We also have a product version of Theorem 3 .

Theorem 5. Let $\left\{\omega^{s, t}\right\}_{s, t \in[0,1]}$ be a family of doubling weights on $\mathbb{T} \otimes \mathbb{T}$, with doubling constants bounded by a constant $C_{\mathrm{dbl}}$ independent of $s, t \in[0,1]$. Suppose the mapping $(s, t) \mapsto \omega^{s, t}$ is measurable. Fix $p$ with $1 \leq p \leq \infty$, and suppose each $\omega^{s, t}$ is in $A_{p}^{d}(\mathbb{T} \otimes \mathbb{T})$ with $A_{p}^{d}(\mathbb{T} \otimes \mathbb{T})$ constant bounded by a constant $V_{p, d}$ independent of $s, t \in[0,1]$. Then the translation-average

$$
\omega(x, y):=\int_{0}^{1} \int_{0}^{1} \omega^{s, t}(x+s, y+t) d s d t
$$

belongs to $A_{p}(\mathbb{T} \otimes \mathbb{T})$, with an $A_{p}$ constant depending only on $p, V_{p, d}$, and $C_{\mathrm{dbl}}$.

Similarly, for $1<p \leq \infty$, if the $A_{p}^{d}(\mathbb{T} \otimes \mathbb{T})$ assumption above is replaced by the assumption that each $\omega^{s, t}$ is in $R H_{p}^{d}(\mathbb{T} \otimes \mathbb{T})$ with the supremum in the defining inequality for $R H_{p}^{d}(\mathbb{T} \otimes \mathbb{T})$ bounded by a constant $V_{p, d}$ independent of $s, t \in[0,1]$, then $\omega(x, y)$ belongs to $R H_{p}(\mathbb{T} \otimes \mathbb{T})$, with $R H_{p}(\omega)$ depending on $p, V_{p, d}$, and $C_{\mathrm{dbl}}$.

In brief, the geometric-arithmetic average of each suitable family of $A_{p}(\mathbb{T} \otimes \mathbb{T})$ weights is also an $A_{p}(\mathbb{T} \otimes \mathbb{T})$ weight, and similarly for $R H_{p}(\mathbb{T} \otimes \mathbb{T})$.

The proof is by iteration of the one-parameter argument given above for Theorem 3.

\section{References}

[1] Buckley, S. M.: Summation conditions on weights. Michigan Math. J. 40 (1993), no. 1, 153-170.

[2] Cruz-Uribe, D. and Neugebauer, C. J.: The structure of the reverse Hölder classes. Trans. Amer. Math. Soc. 347 (1995), no. 8, 2941-2960.

[3] García-Cuerva, J. and Rubio de Francia, J. L.: Weighted norm inequalities and related topics. North-Holland Mathematics Studies 116. North-Holland Publishing Co, Amsterdam, 1985.

[4] Garnett, J.B.: Bounded analytic functions. Pure and Applied Mathematics 96. Academic Press, New York-London, 1981.

[5] Garnett, J. B. and Jones, P. W.: BMO from dyadic BMO. Pacific. J. Math. 99 (1982), no. 2, 351-371.

[6] Grafakos, L.: Classical and modern Fourier analysis. Pearson Education, Upper Saddle River, NJ, 2004.

[7] JAWERTh, B.: Weighted inequalities for maximal operators: linearization, localization and factorization. Amer. J. Math. 108 (1986), no. 2, 361-414.

[8] John, F. And Nirenberg, L.: On functions of bounded mean oscillation. Comm. Pure Appl. Math. 14 (1961), 415-426. 
[9] Johnson, R. And Neugebauer, C. J.: Homeomorphisms preserving $A_{p}$. Rev. Mat. Iberoamericana 3 (1987), no. 2, 249-273.

[10] Jones, P. W.: Factorization of $A_{p}$ weights. Ann. of Math. (2) 111 (1980), no. $3,511-530$.

[11] Lin, K.-C.: Harmonic analysis on the bidisc. Ph.D. thesis, UCLA, 1984.

[12] Muckenhoupt, B.: Weighted norm inequalities for the Hardy maximal function. Trans. Amer. Math. Soc. 165 (1972), 207-226.

[13] Pipher, J. And WARD, L. A.: BMO from dyadic BMO on the bidisc. J. Lond. Math. Soc. (2) 77 (2008), no. 2, 524-544.

[14] Stein, E. M.: Harmonic analysis: Real-variable methods, orthogonality, and oscillatory integrals. Princeton Mathematical Series 43. Monographs in Harmonic Analysis, III. Princeton University Press, Princeton, NJ, 1993.

[15] Treil, S.: $H^{1}$ and dyadic $H^{1}$. In Linear and complex analysis, 179-194. AMS Transl. Ser. 2 226. Amer. Math. Soc., Providence, RI, 2009.

[16] Ward, L. A.: Translation averages of dyadic weights are not always good weights. Rev. Mat. Iberoamericana 18 (2002), no. 2, 379-407.

Recibido: 15 de diciembre de 2009

Jill Pipher

Department of Mathematics

Brown University

Providence, RI 02912, USA

jpipher@math.brown.edu

Lesley A. Ward

School of Mathematics and Statistics

University of South Australia

Mawson Lakes, SA 5095, Australia

lesley.ward@unisa.edu.au

Xiao Xiao

Department of Mathematics

Brown University

Providence, RI 02912, USA

xxiao@math. brown.edu

The first and the third author are supported by the NSF under grant no. DMS0901139. 